Authors statement: The authors realised errors in the calculations related to this study, which impact the conclusions of the work reported here.

\title{
Gas adsorption in amorphous porous boron oxynitride: Grand Canonical Monte Carlo simulations and experimental determination
}

\author{
Ravi B. Shankar, Sofia Marchesini, Erich A. Müller, Camille Petit ${ }^{\star}$
}

Barrer Centre, Department of Chemical Engineering, Imperial College London, South Kensington Campus, Exhibition Road, London SW7 2AZ, United Kingdom

*Corresponding authors:

E-mail: e.muller@imperial.ac.uk; Phone: +44 (0)20 75941569 (Erich A. Müller)

E-mail: camille.petit@imperial.ac.uk; Phone: +44 (0)20 75943182 (Camille Petit) 


\section{Abstract}

Amorphous boron nitride doped with oxygen, boron oxynitride, BNO, is a porous material stable at high pressures and elevated temperatures with potential uses in adsorption-based separation processes at the industrial scale. We present here a molecular model capable of accurately predicting gas sorption in porous BNO solely from the knowledge of the basic experimental characteristics, i.e. overall chemical composition and porosity. With this information, the adsorbent is described atomistically by a complex 3-D pore network built by random packing of nanoflakes. The adsorption may then be evaluated by employing Grand Canonical Monte Carlo with classical forcefields. We report sorption isotherms for $\mathrm{CO}_{2}, \mathrm{~N}_{2}$ and $\mathrm{CH}_{4}$ on $\mathrm{BNO}$ at low (<1 bar) and high ( $0-20$ bar) pressures, across a range of temperatures $(283-313 \mathrm{~K})$, which are well predicted by the molecular model. While the experimental measurement of multi-component isotherms under such conditions is a challenging task, molecular simulations provide predictions without the need of additional information. As an example, $\mathrm{CO}_{2} / \mathrm{N}_{2}$ and $\mathrm{CO}_{2} / \mathrm{CH}_{4}$ binary mixture isotherms, at conditions relevant to post combustion $\mathrm{CO}_{2}$ capture and natural gas sweetening, are computed. Overall, the model provides fundamental insight, which is useful in the design and optimization of porous BNObased adsorbents for molecular separations.

Key words: boron nitride, adsorption, porosity, molecular simulation, Monte Carlo 
Molecular simulations play a pivotal role in the understanding of processes at the atomisticlevel where fluids and solids coexist. ${ }^{1}$ These interfacial systems are the basis of separation processes aimed at addressing ongoing global challenges including, but not restricted to, carbon capture, air pollution, and water purification. Tackling these challenges calls for the development of porous materials, exhibiting robustness and tunable physical-chemical properties, to facilitate separations at the micro- and mesoscale. For these purposes, a wide platform of porous materials are currently under research, which include metal-organic frameworks (MOFs), ${ }^{2-4}$ covalent-organic frameworks (COFs), ${ }^{5-7}$ activated carbon, ${ }^{8-10}$ porous organic polymers, ${ }^{11-13}$ and inorganic compounds such as zeolites. ${ }^{14-16}$ These materials have been employed across a range of applications, such as gas storage,${ }^{17-20}$, drug delivery, ${ }^{21-24}$ water treatment, ${ }^{25} \mathrm{CO}_{2}$ capture, ${ }^{26-29}$ and catalysis. ${ }^{30-33}$

Porous boron oxynitride (BNO) has recently emerged as a prominent member of this material platform. ${ }^{34-40}$ Often denoted as the inorganic counterpart to activated carbon, this material has been employed for facilitating molecular separations, both in gaseous ${ }^{41-43}$ and liquid media. ${ }^{44-}$ ${ }^{47}$ Porous BNO is a disordered 3-D material, in which boron, nitrogen, and oxygen atoms are bonded together in $\mathrm{sp}^{2}$-hybridized hexagonal lattices. These lattices, stacked in random orientations, lack long-range structural order and possess a considerable number of defects, thus giving the material a turbostratic/amorphous morphology. We distinguish porous BNO from other BN materials, such as crystalline hexagonal $B N(h B N)$, owing to the significant proportion of interior-substituted oxygen and its porous and disordered nature. Template-free syntheses, typically involving the reaction of boron- and nitrogen-containing precursors at elevated temperatures $\left(>1000^{\circ} \mathrm{C}\right)$ in the presence of an inert (nitrogen or argon) or ammonia atmosphere, are commonly employed to synthesize porous BNO. $45,48,49$

The tunable chemistry and potentially high specific surface area and pore volume are among the desirable features of this material that have encouraged several groups to pursue studies pertaining to its employment for molecular separations. Marchesini et al. recently developed a template-free synthesis route tailored towards the production of porous BNO and tuning of 
its porosity. ${ }^{34}$ The authors demonstrated experimental tunability of the pore structure from the nano- to the macroscale, which enables the material to be tailored towards a given application. The pore network was directly visualised using 3-D tomography, which provided valuable insight into the mesopore structure of porous BNO.

From a computational perspective, there is presently a lack of accurate molecular simulation models for gas adsorption in BN-based materials, specifically those exhibiting an amorphous morphology. A desirable feature of such models should be the ability to fine-tune the morphology at the nanoscale while retaining the ability to accurately predict single or multicomponent adsorption isotherms at high pressures and elevated temperatures. These models can also be used to gain molecular level insights into the adsorption processes and guide the development of applications. Among the few studies on the topic, the computational work of Lu et al. ${ }^{50}$ is significant. The authors used DFT and grand canonical Monte Carlo simulations to investigate the competitive adsorption of a $\mathrm{CO}_{2} / \mathrm{CH}_{4}$ mixture in porous $\mathrm{BN}$. The substrate size, pore type and electrostatic interactions were found to influence trade-offs between adsorption capacity and selectivity. Other relevant studies have focused on studying the interactions of single component and/or binary mixtures in hBN channels or slit pores ${ }^{51-53}$ and BN nanotubes ${ }^{54-56}$ (BNNTs) by molecular dynamics employing either classical or ab-initio forcefields. These studies have provided insight into the adsorption mechanism in BN-based adsorbents, not least at the advent of widespread interest in the material. However, Kumar et al. ${ }^{57}$, echoed by Di Biase and Sarkisov, ${ }^{58}$ recognized that the slit pore model yields an inaccurate and inadequate description of the complex geometry of porous materials, namely activated carbons. Being the inorganic analogue of activated carbon, this notion is likely true for porous BNO. Further, the underlying oversight or omission in previous studies pertaining to molecular models for BN adsorption has been the lack of synergy with experimental isotherm data for model validation and prediction.

Herein, we present a molecular model to simulate and predict gas sorption in a porous BNO adsorbent, exhibiting a 3-D turbostratic/amorphous morphology. The adsorbent structure 
mimics the complex pore network, previously visualised through scanning transmission electron microscopy (STEM) tomography by Marchesini et $a l^{34}$, in an experimentally synthesized porous BNO sample. This approach relies on the random stacking and dispersion of BNO nanoflakes to generate a 3-D porous network. A similar approach had been suggested by Salih and Müller ${ }^{59}$ and has been previously employed for activated carbons by Kumar et al. ${ }^{57}$, and later by Di Biase and Sarkisov. ${ }^{58}$ Through this method, we introduce a random porosity, essentially an additional layer of modelling complexity when compared to the slit pore model of adjacent nanosheet layers or stacked BN nanosheets with a constant $d$-spacing (akin to crystalline $\mathrm{hBN}$ ). In addition to the morphology, the chemical composition of the simulated adsorbent was tailored and matched to that obtained for the experimental porous BNO sample via X-ray photoelectron spectroscopy (XPS). An underlying highlight of our model is that the chemical composition of the simulated BNO adsorbent can be easily modified to match experimental measurements. The synthesized porous BNO sample was characterized extensively to provide insight into the chemistry, structure, porosity and morphology during model development. Gas sorption of a range of adsorbates $\left(\mathrm{N}_{2}, \mathrm{CO}_{2}\right.$, and $\left.\mathrm{CH}_{4}\right)$ at both low ( $<1$ bar) and high (up to 20 bar) pressures, over a range of temperatures ( $283-313 \mathrm{~K}$ ), was modelled and predicted through Grand Canonical Monte Carlo ${ }^{60}$ (GCMC) simulations using the $M e d e A^{\circledR 61}$ software. There was no attempt to force-fit the model predictions (e.g. by modifying the solid-fluid potentials). The predictions were validated against experimental gas sorption measurements for a porous BNO sample, exhibiting similar structure and chemistry, under the same pressure and temperature conditions. The simulation model predicted the shape and magnitude of the sorption isotherms for all of the tested adsorbates, under the range of conditions investigated, with minimal deviation from experimental measurements. We envision such a computational model to be a valuable prediction-based tool to be utilized either prior to, or in tandem with, experimental testing of porous BN-based adsorbents. Overall, we claim that the current study brings an integrated molecular engineering approach that delivers practical insights into the design and optimization of porous BN-based adsorbents. 


\section{Results and discussion}

We synthesized porous BNO following Marchesini et al. ${ }^{34}$, whose approach relies on a bottomup pyrolytic synthesis at elevated temperatures using a mixture of boric acid, melamine and urea. To gain insight into the morphology and structure of the material in comparison to $\mathrm{hBN}$, we collected high resolution transmission electron microscopy (HRTEM) and scanning electron microscopy (SEM) images, as well as powder X-ray diffraction (XRD) patterns (Figures 1 and S1). Both techniques confirm the low degree of crystallinity of porous BNO as opposed to hBN. The HRTEM images depict the limited range of stacked 2D layers in porous BNO (Figure 1a), which contrasts with the long-range crystalline domains observed in hBN (Figure S1a). The XRD patterns confirm the turbostratic nature of the material with only broad peaks at $2 \theta$ values of $26^{\circ}$ and $44^{\circ}$, corresponding to the (002) and (100) planes, respectively (Figure 1b). ${ }^{62}$ This contrasts with the sharp peaks for $\mathrm{hBN}$ that indicate long-range 3D/graphitic order (Figure S1b). The d-spacing calculated from the TEM images and XRD peak (002) for $\mathrm{hBN}$ and porous $\mathrm{BNO}$ is $3.4 \AA$ and $3.5 \AA$, respectively. The d-spacing in porous BNO is only relevant to the pseudo-crystalline and turbostratic regions of the material. The SEM images depict a densely packed pore structure in porous BNO (Figure S2). These analyses indicate the formation of an amorphous/turbostratic porous BNO.

To characterize the structural features of the material at the micro/nano-scale, we analyzed the specific surface area and porosity through nitrogen sorption at $77 \mathrm{~K}$ (Figures 1c and Figure $\mathrm{S} 1 \mathrm{c}$ for isotherms, Table S1 for key textural parameters). The porous BNO sample displayed a BET equivalent specific surface area of $1924 \mathrm{~m}^{2} \mathrm{~g}^{-1}$ and total pore volume of $1.20 \mathrm{~cm}^{3} \mathrm{~g}^{-1}$, which is considerably larger than those for $\mathrm{hBN}\left(3 \mathrm{~m}^{2} \mathrm{~g}^{-1}\right.$ and $\left.0.006 \mathrm{~cm}^{3} \mathrm{~g}^{-1}\right)$. The type I/IV isotherm with a type $\mathrm{H} 3 / \mathrm{H} 4$ hysteresis loop observed for porous $\mathrm{BNO}$ indicates the presence of slit-shaped micro- and mesopores. The high specific surface area and porosity of porous BNO plays an essential role in interfacial phenomena, enhancing access to adsorption sites. This is particularly important in gas phase molecular separations and storage applications, like the one we investigate herein. 
a

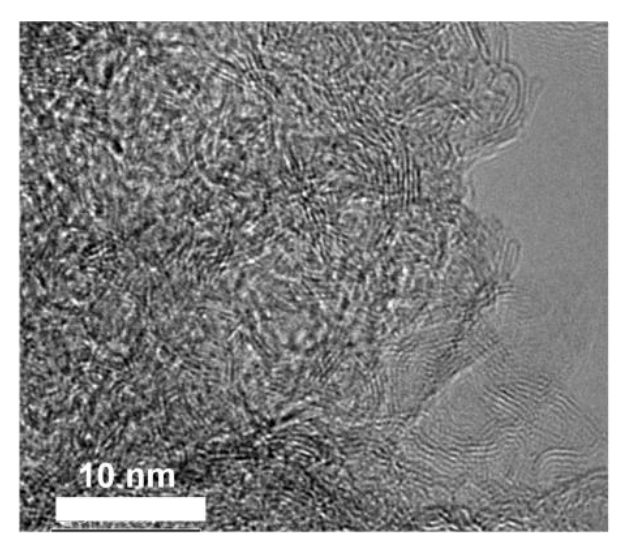

C

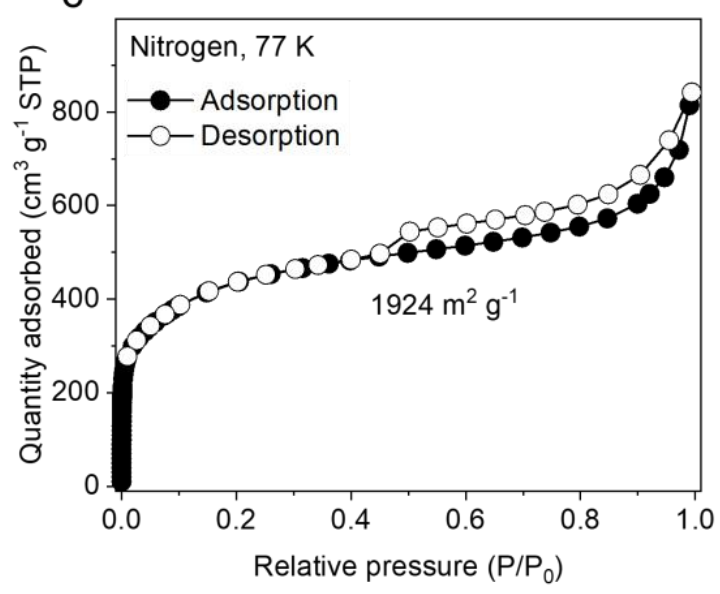

b

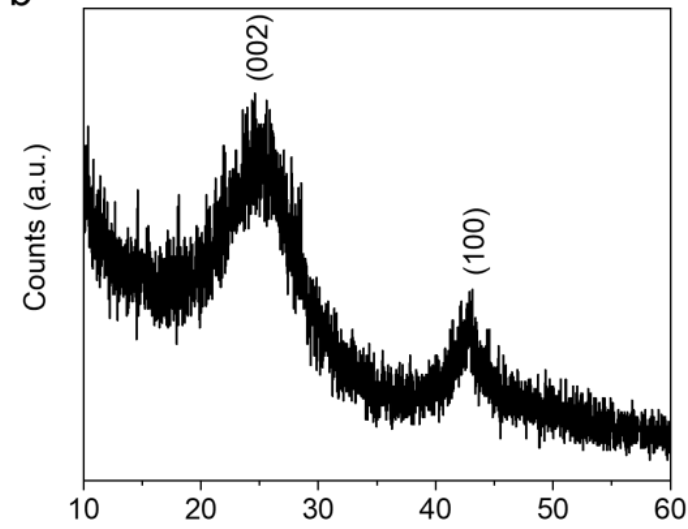

d

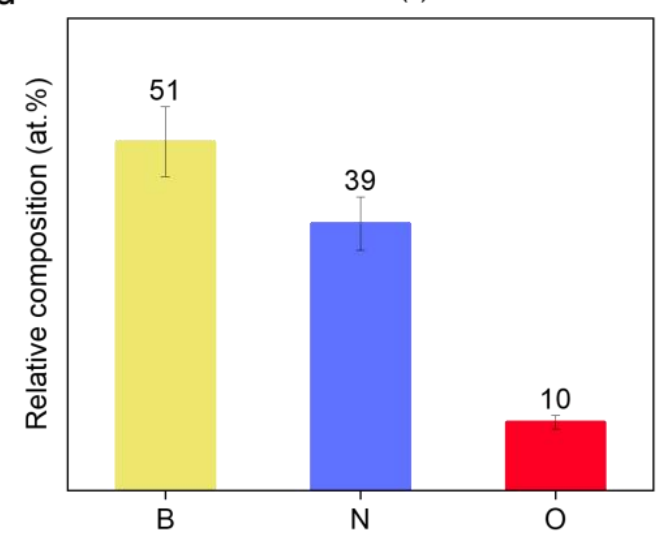

Figure 1 I Structure and chemistry of the porous BNO sample. (a) HRTEM image of the as-synthesized porous BNO sample, highlighting the disordered morphology through the absence of large crystalline domains, (b) Powder XRD pattern of porous BNO, (c) $\mathrm{N}_{2}$ sorption isotherms for porous BNO measured at $77 \mathrm{~K}$, (d) Relative atomic composition of porous BNO as obtained through XPS analysis.

To gain further insight into the surface chemistry and relative atomic composition of porous BNO, we collected survey and high resolution core level spectra through X-ray photoelectron spectroscopy (XPS). The relative atomic composition of porous BNO and hBN are shown in Figures $1 \mathrm{~d}$ and S1d, respectively. We note that while XPS is a surface technique and may not be representative of the bulk sample, it still provides a good indication of the composition. The survey spectrum and peak deconvolution of the $\mathrm{B} 1 \mathrm{~s}, \mathrm{~N} 1 \mathrm{~s}$ and $\mathrm{O} 1 \mathrm{~s}$ high resolution core level 
spectra is presented in Figure S3. The formation of a BN-based material is confirmed through the fitted core level spectra of the B $1 s$ and $\mathrm{N} 1 s$ and show the presence of B-N bonds (191.0 eV for B $1 s$ and $398.5 \mathrm{eV}$ for N 1s, Figure S3). ${ }^{63}$ We also observed shake-up satellite peaks in the B $1 s$ and N 1 s core level spectra for porous BNO (Figure S3), which points to the formation of a $\mathrm{sp}^{2}$-hybridized hexagonal $\mathrm{BN}$ phase. ${ }^{64,65}$ The analysis indicates a significant proportion (10 at.\%) of oxygen in porous BNO (Figure 1d) with a peak at $533.1 \mathrm{eV}$ (Figure S3). We attribute this peak to boron oxynitride $\left(B-\mathrm{O}_{x}-\mathrm{N}_{3-\mathrm{x}}\right)$ species, which stems from the in-plane substitution of nitrogen atoms with oxygen atoms into the BN lattice, as described in prior studies. ${ }^{35,48}$ Oxygen atoms were observed in hBN and are most likely related to edge hydroxyl groups (Figure S1d). The carbon content in the porous BNO sample was comparatively much lower ( 2 at. \%). It was excluded from the analysis as it was linked to the presence of adventitious carbon impurities. The chemistry and bonding types of porous BNO was further characterized through Raman spectroscopy and Fourier transform infrared spectroscopy (FTIR) (Figures S4 and S5). Both porous BNO and hBN exhibit a strong Raman peak at $\sim 1370 \mathrm{~cm}^{-1}$, corresponding to the counter-phase $B-N E_{2 g}$ vibration mode within the $B N$ structure (Figure S5) ${ }^{66}$ We observed the two characteristic IR bands of BN at $\sim 1380 \mathrm{~cm}^{-1}$ (inplane B-N transverse stretching) and $\sim 800 \mathrm{~cm}^{-1}$ (out-of-plane B-N-B bending) in both samples, ${ }^{67}$ while no B-O bands, typically observed at $\sim 1000 \mathrm{~cm}^{-1}$, are noted (Figure S6). ${ }^{36,48}$

We developed the computational model of the porous BNO adsorbent within the MedeA $A^{\circledR 61}$ environment using the structural and chemical analyses of the as-synthesized porous BNO material in Figure 1. The overall computational strategy is depicted in Figure 2 and can be summarized in the following stages (see Computational Model Development section for full details). The unit building block is a BNO nanoflake consisting of 24 atoms (Figure 3a). The chemical composition of this BNO nanoflake was tailored to match the experimental relative atomic composition of the synthesized porous BNO, obtained through XPS. The BNO nanoflake building blocks were then inserted into a unit cell with side dimension of $26.343 \AA$, avoiding overlapping of the BNO units. ${ }^{68}$ 


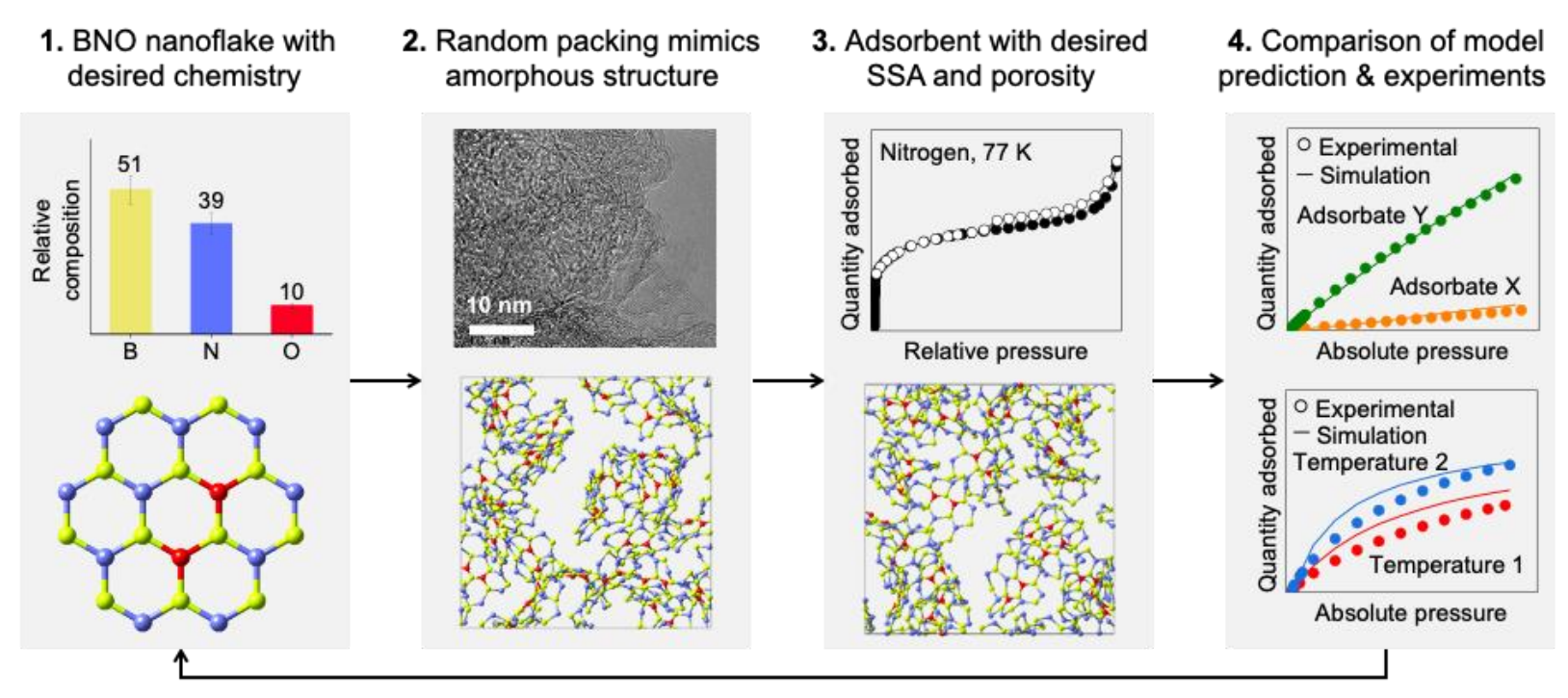

Figure 2 I Development of the computational porous BNO adsorbent model. Schematic depicting the overall computational strategy employed herein. The strategy is based on an integrated molecular engineering approach towards porous adsorbent screening for gas separation/storage applications. In Stage 1, one tailors the chemistry of the unit building block to experimental (XPS) measurements. The amorphous structure of porous BNO is mimicked through random packing in Stage 2. The structure, namely the specific surface area and porosity, of the porous BNO adsorbent can be tuned to match experimental gas sorption measurements in Stage 3. Finally, Stage 4 entails running model simulations of single component or multi component gas sorption isotherms and comparing to experimental measurements. A circular process is envisaged herein to allow one to further refine the model in the event of discrepancies with experimental measurements.

No bridges were added between the structures and they were artificially fixed in space within the cell at time $t=0$ seconds. A canonical (constant density and temperature) molecular dynamics simulation is performed at an arbitrarily high temperature (3000 K) while keeping the dispersion interactions between the nanoflakes disabled to achieve a random packing and preventing agglomeration. Naturally, a number of realizations of this structure may be obtained by different spatial placement of the basic units and/or longer equilibration times. Different realizations of the BNO adsorbent structure were obtained using the same molecular dynamics simulation conditions (see Computational Model Development section for full details), but with different equilibration times. The simulations showed that the densities of 
different BNO adsorbent realizations are indeed independent of the different spatial placements of the basic units and/or longer equilibration times. The simulated porous BNO adsorbent model is shown in Figure $3 b$. The nanoflakes were randomly packed to mimic the turbostratic/amorphous structure of porous BNO shown in Figure 1. The structure and morphology in Figure 3b agree with the 3D tomographic reconstruction of the pore network of porous BNO previously reported by Marchesini et al. ${ }^{34} \mathrm{~A}$ key highlight of this model is the ability to tune both the structure and chemistry concomitantly to match experimental values, as one can vary at will the original packing density and/or the chemical composition of the flakes. Further refinement of the model can be made by introducing polydispersity in the size shape and/or curvature of the flakes. The textural parameters of the simulated adsorbent, namely the accessible surface area and total pore volume, were obtained using the Poreblazer software developed by Sarkisov and Harrison using an $\mathrm{N}_{2}$ probe molecule. ${ }^{69}$ The accessible surface area and total pore volume for the simulated porous BNO adsorbent was determined to be $1427 \mathrm{~m}^{2} \mathrm{~g}^{-1}$ and $1.00 \mathrm{~cm}^{3} \mathrm{~g}^{-1}$, respectively, which show reasonable agreement with the corresponding experimental values (specific surface area of $1924 \mathrm{~m}^{2} \mathrm{~g}^{-1}$ and total pore volume of $1.20 \mathrm{~cm}^{3} \mathrm{~g}^{-1}$ ) (Figure $3 \mathrm{c}$ ). Surface chemistry is also critically important when gas sorption applications are considered. 
a
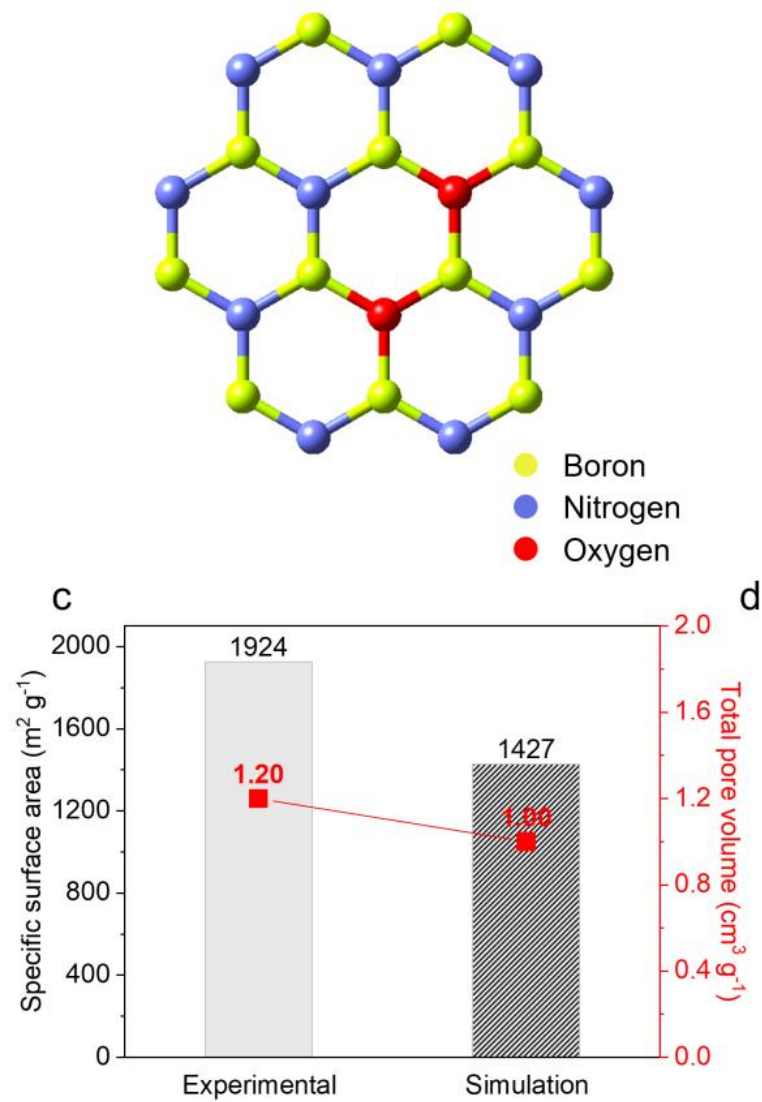

b
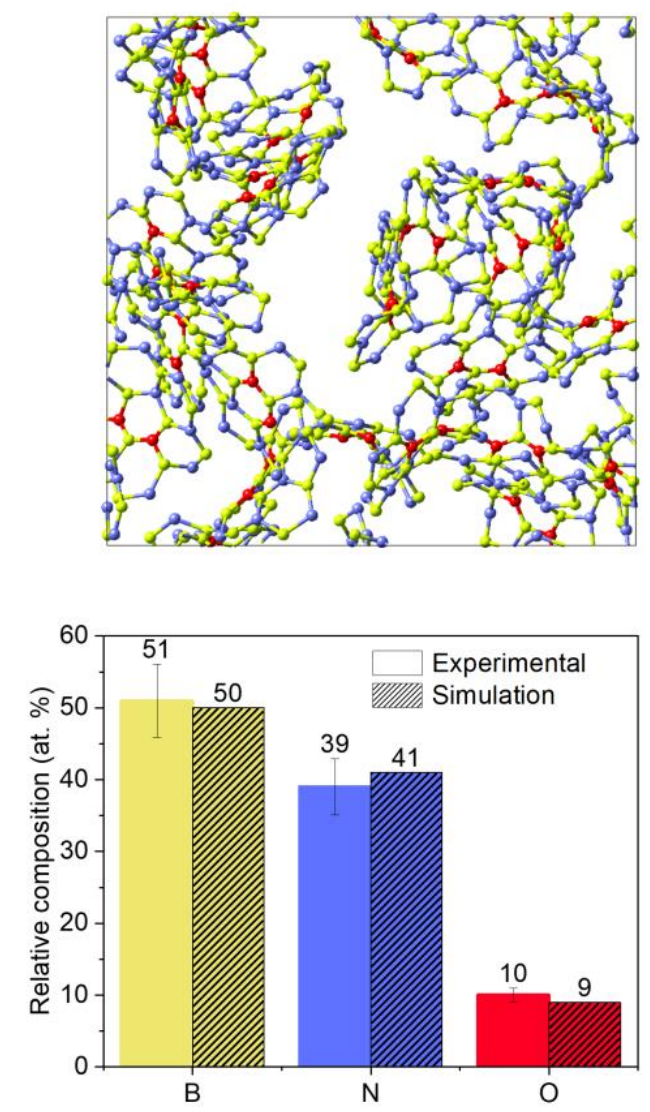

Figure 3 I Characterization of the computational porous BNO adsorbent model in comparison to experimental data. (a) Schematic of the BNO nanoflake used as the building block to construct the porous BNO adsorbent with an amorphous/turbostratic morphology. The relative atomic composition of the nanoflake is tuned to match the experimental values obtained through XPS analysis. The yellow, blue, and red atoms denote boron, nitrogen and oxygen, respectively, (b) The porous BNO adsorbent unit cell, (c) Comparison of the specific surface area and total pore volume of the experimental and simulated porous BNO adsorbent, (d) Comparison of the simulated relative atomic composition compared with the experimental values obtained through XPS measurements.

Having developed and characterized the porous BNO adsorbent model, we next conducted GCMC simulations for $\mathrm{N}_{2}, \mathrm{CO}_{2}$ and $\mathrm{CH}_{4}$ at low ( $<1$ bar) and high pressures ( $0-20$ bar) at 
room temperature $(298 \mathrm{~K})$. Classical force fields were employed to characterize the fluid-fluid and fluid-solid interactions (see Computational Model Development). The simulated isotherms and adsorption capacities for both low and high pressure gas sorption were compared to the experimental results of Marchesini et al. ${ }^{34}$, obtained for the same material under identical conditions. The error between the model predicted and experimental adsorption capacities for each isotherm was quantified through the root mean square deviation (RMSD) measure, defined as the square root of the mean square error (MSE) (see Tables S2-S8 for error analysis). The RMSD and MSE are measures of accuracy between values predicted by a model or an estimator to the observed values; the closer the MSE and RMSD to zero, the smaller the residuals and stronger the fit to experimental data. ${ }^{70}$

We first investigated the model adsorption behaviour in the Henry's law region at low pressures (< 1 bar, $298 \mathrm{~K}$ ) and loading densities, where adsorbate-adsorbate interactions are negligible. The model is capable of accurately modelling the linear adsorption behaviour of both $\mathrm{N}_{2}$ and $\mathrm{CO}_{2}$ at $298 \mathrm{~K}$ in the Henry's law region, quantified through a coefficient of determination of $R^{2}=0.9953$ and 0.9976 , respectively (Figure 4a). The total RMSD for the $\mathrm{N}_{2}$ and $\mathrm{CO}_{2}$ isotherms over the low pressure range $(<1$ bar, $298 \mathrm{~K})$ was 0.03 in both cases, indicating a high degree of accuracy in the isotherm predictions. To demonstrate the versatility of the model to a range of adsorbates, low pressure $\mathrm{CH}_{4}$ sorption isotherms at $298 \mathrm{~K}$ were also modelled accurately with an $\mathrm{R}^{2}$ value of 0.99 and RMSD of 0.10 (Figure S5). At 1 bar and $298 \mathrm{~K}$, the model predicts a $\mathrm{CO}_{2}$ uptake of up to $1.55 \mathrm{mmol} \mathrm{g}^{-1}$, which is in good agreement with the experimentally measured value of $1.50 \mathrm{mmol} \mathrm{g}^{-1}$ of $\mathrm{CO}_{2}$ for porous $\mathrm{BNO}$.

High pressure gas sorption isotherms for $\mathrm{N}_{2}$ and $\mathrm{CO}_{2}(0-20$ bar, $298 \mathrm{~K})$ were modelled and compared to the experimental results of Marchesini et al. (Figure 4b). ${ }^{34}$ The simulations are capable of capturing the shape of the $\mathrm{N}_{2}$ and $\mathrm{CO}_{2}$ isotherms and accurately predicting the associated sorption capacities at higher pressures beyond the Henry's law region, as illustrated by the close overlap with the experimental data (RMSD $=0.30$ and 0.53 for the $\mathrm{N}_{2}$ and $\mathrm{CO}_{2}$ sorption isotherms, respectively). At 20 bar and $298 \mathrm{~K}$, the model predicts a $\mathrm{CO}_{2}$ and 
$\mathrm{N}_{2}$ uptake of $8.03 \mathrm{mmol} \mathrm{g}^{-1}$ and $1.41 \mathrm{mmol} \mathrm{g}^{-1}$, respectively, which aligns well with the experimentally measured values of $8.16 \mathrm{mmol} \mathrm{g}^{-1}$ and $1.35 \mathrm{mmol} \mathrm{g}^{-1}$, respectively. The results indicate that the model is capable of accounting for the effects of physisorption at higher pressures (Figure 4b).

As a potential adsorbent screening tool, one would expect such a simulation model to deliver accurate results across a range of pressures, but also at different adsorption temperatures. As such, high pressure $\mathrm{CO}_{2}$ sorption in porous $\mathrm{BNO}$ was also simulated at $283 \mathrm{~K}$ and $313 \mathrm{~K}$ and compared to experimental data (Figure 4c). Albeit with slightly larger overpredictions compared to the experimental isotherms $(\mathrm{RMSD}=1.00$ and 1.17 at $283 \mathrm{~K}$ and $313 \mathrm{~K}$, respectively), the model is able to capture the trends in adsorption capacities. Marchesini et al. ${ }^{34}$ experimentally determined the heat of adsorption for $\mathrm{CO}_{2}$ in porous $\mathrm{BNO}$ to be approximately $19 \mathrm{~kJ} \mathrm{~mol}^{-1}$ for high pressure $\mathrm{CO}_{2}$ sorption ( $0-20$ bar, $298 \mathrm{~K}$ ), which aligned with the theoretical calculation for $\mathrm{CO}_{2}$ physisorption on a $\mathrm{BN}$ sheet. ${ }^{71}$ The heat of adsorption obtained through GCMC for $\mathrm{CO}_{2}$ sorption under the same conditions is presented in Figure $4 \mathrm{~d}$ and aligns with the experimental values of Marchesini et al. ${ }^{34}$ with a slight overestimation.

This discrepancy could originate from knock-on effects of the solid-fluid interaction assumptions. We have assumed that the adsorbent-adsorbate interactions (e.g. B-CO $\mathrm{CO}_{2}$ or$\mathrm{N}_{2}$ ) can be interpolated from the knowledge of the force field parameters for individual atoms (e.g. carbon), and the adsorbates (i.e. $\mathrm{CO}_{2}$ and $\mathrm{N}_{2}$ ). However, this is usually not true as such interactions need to be fine-tuned, typically to the low pressure isotherms in the Henry's law region, where only the interactions of a few adsorbates with the surface is present. ${ }^{72}$ Another source of error could stem from inaccuracies in the force-field parameters of the BNO adsorbent system itself. For an amorphous/turbostratic morphology, as modelled for the porous BNO adsorbent, the finite size of the flakes, the edge effects, the polarizability of the atoms and the overall solid morphology cause variations in the solid-solid and solid-fluid interactions, which we have not accounted for. There is scope for developing accurate tailored forcefields, based on DFT or quantum mechanical methods, for porous BNO and gaseous 
adsorbates..$^{73-75}$ To gain insight into the adsorption mechanism, the configurations of the $\mathrm{CO}_{2}$ adsorbate on porous BNO were mapped and are presented for low and high pressure sorption in Figures $4 \mathrm{e}$ and $4 \mathrm{f}$, respectively. The configurations of $\mathrm{N}_{2}$ on the $\mathrm{BNO}$ adsorbent are presented in Figure S6. In all cases, no preferential adsorptions sites were identified for $\mathrm{CO}_{2}$ or $\mathrm{N}_{2}$ at the oxygen or other atoms.

a

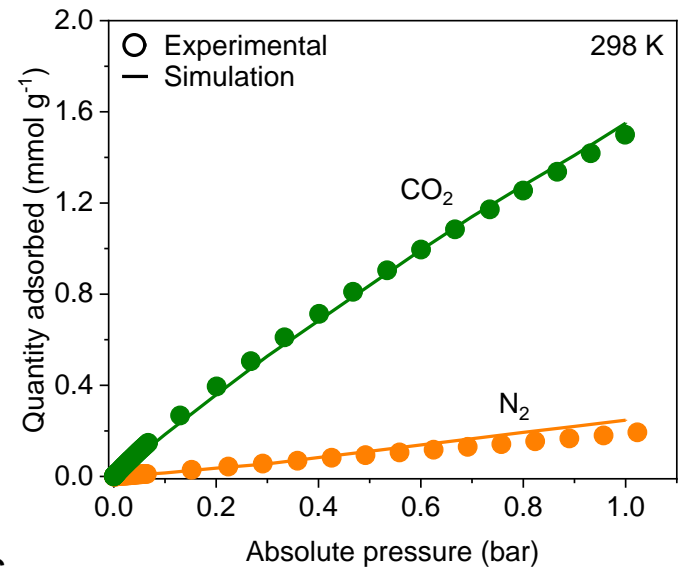

c

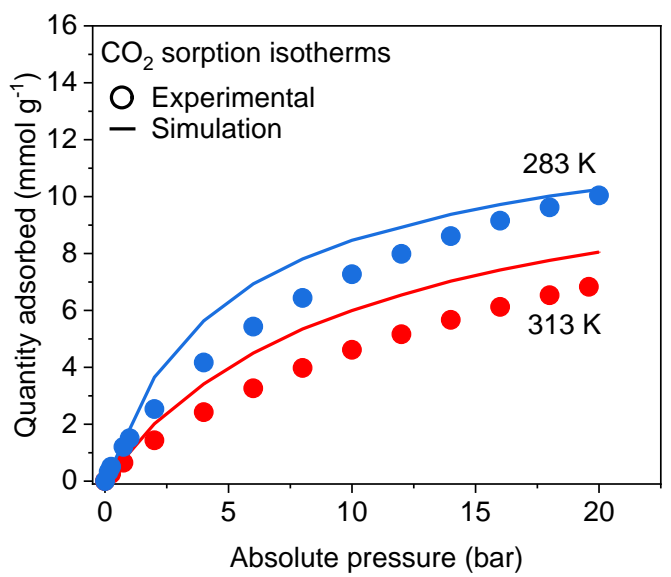

e

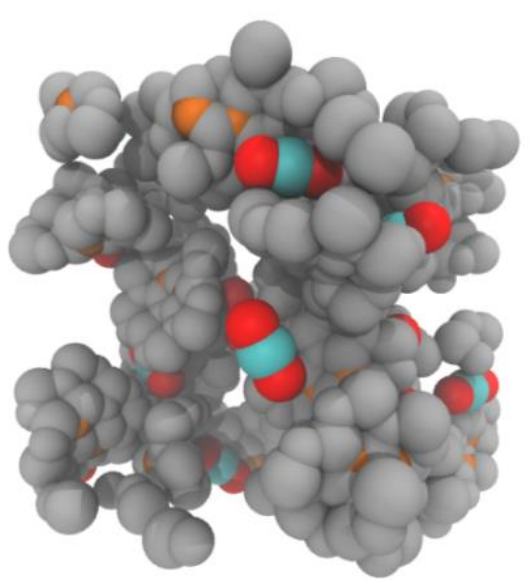

Low pressure $\mathrm{CO}_{2}$ sorption simulation b

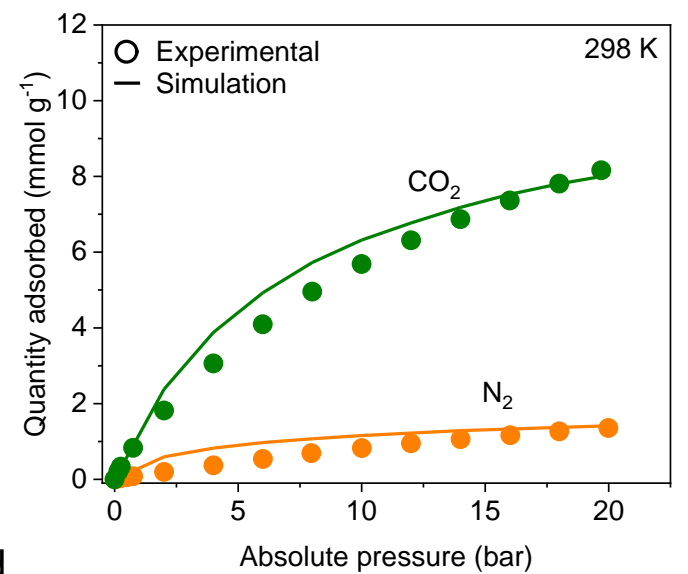

d

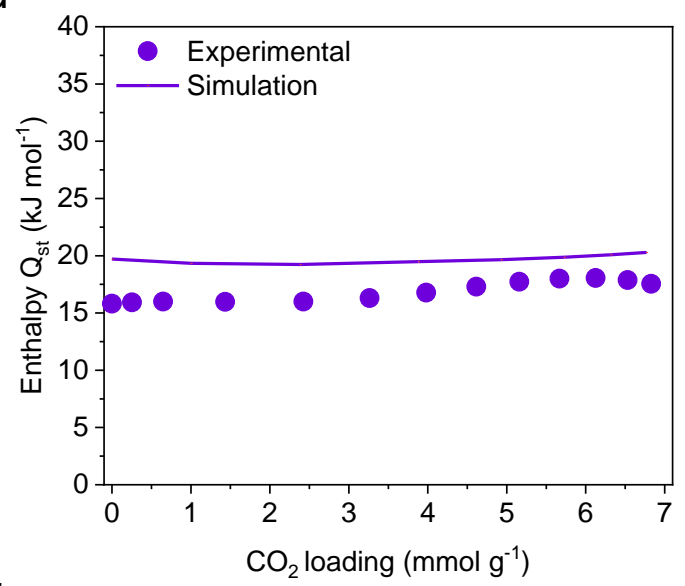

$f$

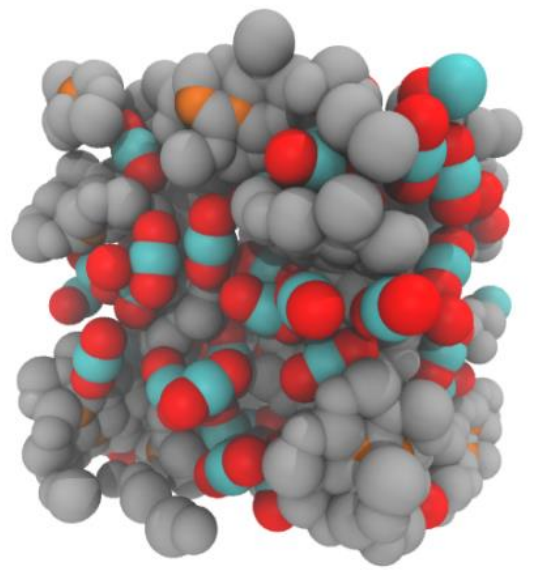

High pressure $\mathrm{CO}_{2}$ sorption simulation 
Figure $4 \mathrm{I}$ Model validation against experimental $\mathrm{N}_{2}$ and $\mathrm{CO}_{2}$ adsorption isotherms at low and high pressures. Comparison of the experimental and simulated $\mathrm{N}_{2}$ and $\mathrm{CO}_{2}$ adsorption isotherms at (a) low pressures ( $<1$ bar) and (b) high pressures $(0-20$ bar) at 298 K. (c) Investigation of model sensitivity to adsorption temperature - comparison of the experimental and predicted high pressure $\mathrm{CO}_{2}$ adsorption isotherms at $283 \mathrm{~K}$ and $313 \mathrm{~K}$, (d) Model predicted heat of adsorption for high pressure $\mathrm{CO}_{2}$ sorption compared to experimental values obtained by Marchesini et al. ${ }^{34}$. Snapshots of equilibrium configurations of the $\mathrm{CO}_{2}$ (red-cyan-red) adsorbate in the porous BNO simulation box at (e) low pressure $(<1$ bar) and (f) high pressure $(0-20 \mathrm{bar})$ at $298 \mathrm{~K}$. Solid atoms are shown in grey with the exception of oxygen atoms which are highlighted in orange.

As an example of how the model can be further employed, we predicted adsorption isotherms and selectivities for binary mixtures of $\mathrm{CO}_{2} / \mathrm{CH}_{4}$ and $\mathrm{CO}_{2} / \mathrm{N}_{2}$. Both separations are directly relevant to the energy sector in the context of natural gas sweetening and post combustion carbon capture, respectively. Isotherms were calculated at the typical pressures and temperatures in such processes: $0-1$ bar and $323-398 \mathrm{~K}$ for post combustion $\mathrm{CO}_{2}$ capture from flue gas ${ }^{76,77}$ and $68-75$ bar and $283-313 \mathrm{~K}$ for natural gas sweetening ${ }^{58}$ (Figure 5). In the context of $\mathrm{CO}_{2}$ capture from flue gas, porous $\mathrm{BNO}$ exhibited $\mathrm{CO}_{2}$ and $\mathrm{N}_{2}$ adsorption capacities of $0.26-0.90 \mathrm{mmol} \mathrm{g}^{-1}$ and $0.08-0.18 \mathrm{mmol} \mathrm{g}^{-1}$, respectively, with $75-83 \%$ selectivity to $\mathrm{CO}_{2}$ over a temperature range of $323-398 \mathrm{~K}$ at 1 bar pressure (Figure 5a). The $\mathrm{CO}_{2}$ and $\mathrm{N}_{2}$ adsorption capacities decreased by $70 \%$ and $55 \%$ as the operation temperature was increased from $323 \mathrm{~K}$ to $398 \mathrm{~K}$. With regards to natural gas sweetening, porous BNO exhibited $\mathrm{CO}_{2}$ and $\mathrm{CH}_{4}$ adsorption capacities of $9.10-10.63 \mathrm{mmol} \mathrm{g}^{-1}$ and $3.21-3.23$ mmol $\mathrm{g}^{-1}$, respectively, with $74-77 \%$ selectivity to $\mathrm{CO}_{2}$ over a temperature range of $283-$ $313 \mathrm{~K}$ at 75 bar pressure (Figure $5 \mathrm{~b}$ ). Above $\sim 10$ bar, the $\mathrm{CO}_{2}$ and $\mathrm{CH}_{4}$ simulated isotherms begin to plateau to a constant adsorption capacity that appears to be virtually independent of pressure. The adsorption capacities and selectivities predicted by the model for $\mathrm{CO}_{2} / \mathrm{N}_{2}$ separation from flue gas streams is on par with those reported for activated carbons under similar conditions. ${ }^{78}$ 
a

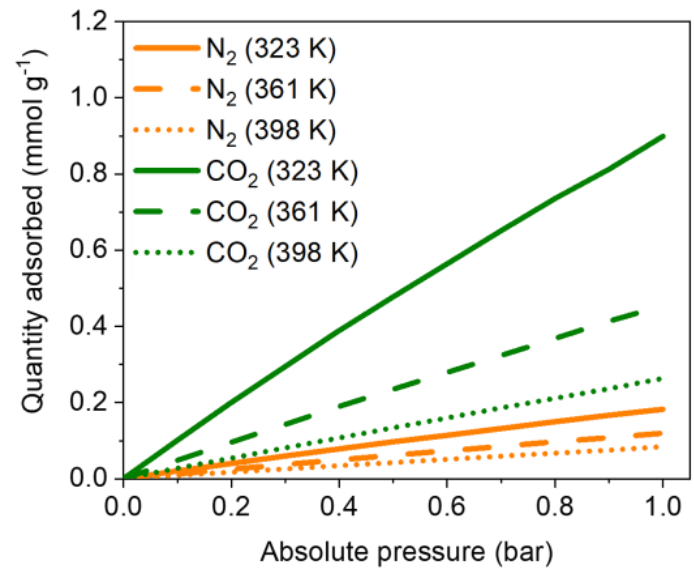

b

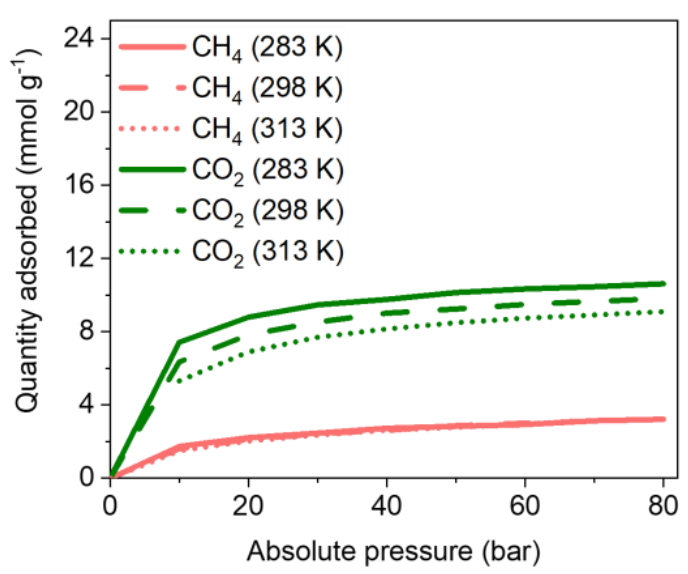

Figure $5 \mathrm{I}$ Model isotherms for binary mixtures of $\mathrm{CO}_{2} / \mathrm{N}_{2}$ for post combustion $\mathrm{CO}_{2}$ capture from flue gas and $\mathrm{CO}_{2} / \mathrm{CH}_{4}$ for natural gas sweetening. (a) Predicted adsorption isotherms for a binary mixture of $\mathrm{CO}_{2} / \mathrm{N}_{2}$ over a pressure range of $0-1$ bar and a temperature range of $323-398 \mathrm{~K}$ in the context of $\mathrm{CO}_{2}$ from flue gas, (b) Predicted adsorption isotherms for a binary mixture of $\mathrm{CO}_{2} / \mathrm{CH}_{4}$ over a pressure range of $0-80$ bar and a temperature range of $283-313 \mathrm{~K}$ in the context of natural gas sweetening. Note the isotherms for $\mathrm{CH}_{4}$ at $298 \mathrm{~K}$ and $313 \mathrm{~K}$ directly overlaps with that at $283 \mathrm{~K}$.

\section{Conclusions}

We present a molecular model that can be used to predict gas sorption in porous BNO. The complex pore geometry and amorphous morphology of porous BNO is modelled through random packing of structural nanoflakes within a simulation box, with the textural parameters and chemical composition tailored to experimental data. The underlying highlight of this approach is the development of the model in tandem with experimental characterization to achieve an accurate representation of the synthesised material. Moreover, the model offers extensive tunability with regards to the porosity, pore size distribution and chemistry, allowing one to tailor the model according to the sample. Model predictions, obtained through Grand Canonical Monte Carlo simulations for $\mathrm{CO}_{2}, \mathrm{~N}_{2}$ and $\mathrm{CH}_{4}$ sorption isotherms, at low (< 1 bar) and high ( $0-20$ bar) pressures exhibited good agreement with experimentally measured isotherms under the same conditions, with minor discrepancy likely resulting from our 
assumptions with respect to the force fields representing the solid-fluid interactions. The chemical heterogeneity of the BN, expressed in terms of the existence of oxygenated sites, did not seem to account for preferential adsorptions sites. We envision such a model to be employed concurrently with experimental adsorption studies to deliver practical insights into the design and optimization of porous BN-based adsorbents for molecular separation.

\section{Acknowledgements}

The authors would like to thank Terrence Crombie for his technical and IT support, Benoît Minisini, David Reith, René Windiks and the Materials Design support team for assisting with software related issues during the early stages of model development, and Kagiso Bikane for his fruitful discussions and insights during the computational model development. The authors would like to acknowledge the funding from EPSRC through the Doctoral Partnership fund (1855454), which made this research possible, as well as ERC Starting Grant THEIA (Project Number: 850624). E.A.M. acknowledges the support from EPSRC through research grants to the Molecular Systems Engineering group (grant nos. EP/E016340, EP/J014958 and EP/R013152)

\section{Declaration}

The authors declare that there are no competing financial interests.

\section{Methods}

Synthesis of porous BNO. In a typical synthesis, boric acid $\left(\mathrm{H}_{3} \mathrm{BO}_{3}, \mathrm{ACS}\right.$ reagent, $99.0 \%$, Sigma-Aldrich), urea $\left(\mathrm{CH}_{4} \mathrm{~N}_{2} \mathrm{O}\right.$, molecular biology grade, Sigma-Aldrich), and melamine $\left(\mathrm{C}_{3} \mathrm{H}_{6} \mathrm{~N}_{6}\right.$, ACS reagent, $99.0 \%$, Sigma-Aldrich), with a 1:1 molar ratio of boric acid to melamine and 1:5 molar ratio of boric acid to urea were mechanically mixed together and finely ground for 5 minutes in an agate mortar to form a homogeneous powder. The finely ground powder was subsequently transferred to an alumina boat crucible, which was placed in a horizontal tubular furnace. The sample was initially degassed at ambient temperature for 3 hours under an inert nitrogen atmosphere (flow rate of $250 \mathrm{~cm}^{3} \mathrm{~min}^{-1}$ ). Once the degas was complete, the 
nitrogen flow rate was decreased to $50 \mathrm{~cm}^{3} \mathrm{~min}^{-1}$, and the sample was heated from ambient temperature to $1050{ }^{\circ} \mathrm{C}$ with a ramp rate of $10{ }^{\circ} \mathrm{C} \mathrm{min}$. . This steady-state temperature was maintained for 3.5 hours, after which the samples were allowed to naturally cool to room temperature, whilst maintaining the same nitrogen flow rate. Upon completion of the synthesis, a porous white powder was obtained, which we refer to as porous BNO.

\section{Materials characterization}

Fourier transform infrared spectroscopy (FT-IR). The samples were first ground to a powder using an agate mortar. Subsequently, the spectra were obtained in the range of 500 - $4000 \mathrm{~cm}^{-1}$ using a Perkin-Elmer Spectrum 100 FT-IR spectrometer equipped with an attenuated total reflectance (ATR) accessory.

X-ray photoelectron spectroscopy (XPS) was employed to determine the relative elemental composition of the samples and the chemical states of the elements was conducted using a Thermo Scientific K-Alpha ${ }^{+}$X-ray Photoelectron Spectrometer equipped with a MXR3 Al K $\alpha$ monochromated X-ray source $(\mathrm{h} v=1486.6 \mathrm{eV})$. The samples were initially ground and mounted onto an XPS sample holder using a small rectangular piece of conductive carbon tape. The X-ray gun power was set to $72 \mathrm{~W}(6 \mathrm{~mA}$ and $12 \mathrm{kV})$. Survey scans were acquired using $200 \mathrm{eV}$ pass energy, $0.5 \mathrm{eV}$ step size and $100 \mathrm{~ms}$ (50 ms $\times 2$ scans) dwell times. All of the high resolution core level spectra $(\mathrm{B} 1 \mathrm{~s}, \mathrm{~N} 1 \mathrm{~s}$, and $\mathrm{O} 1 \mathrm{~s})$ were obtained using a $20 \mathrm{eV}$ pass energy and $0.1 \mathrm{eV}$ step size. The results were analysed using the Thermo Avantage data analysis program. Any charging effect in the spectra was mitigated by using a dual-beam flood gun that uses the combination of low energy electrons and argon ions.

Powder X-ray diffraction (XRD) was performed using a PANalytical X'Pert Pro X-ray diffractometer in reflection-transmission mode with a spinning stage (2 revolutions/second). An anode voltage of $40 \mathrm{kV}$ and emission current of $20 \mathrm{~mA}$ were chosen as the operating conditions using a monochromatic $\mathrm{Cu}-\mathrm{K} \alpha$ radiation source $(\lambda=1.54178 \AA)$. The X'Celerator silicon strip detector was used in the diffractometer. The interplanar $d_{(002)}$-spacing was calculated from the powder XRD patterns using Bragg's law. 
Scanning electron microscopy (SEM) and transmission electron microscopy (TEM) were used to evaluate the morphology of the samples using a scanning electron microscope (SEM, Leo Gemini 1525, Zeiss) in secondary electron mode (InLens detector) at $5 \mathrm{kV}$. The samples were ground, deposited on carbon tape, and coated with $20 \mathrm{~nm}$ of chromium to reduce charging in the microscope. Transmission electron microscopy imaging was collected on a JEOL 2100FX microscope. TEM and scanning TEM imaging were carried out on an FEI Titan 80-300 Cs image-corrected microscope, operated at 80 and $300 \mathrm{kV}$. Sample preparation was performed by dispersing porous BNO powder in ethanol and drop depositing the supernatant on a holey carbon copper grid.

Nitrogen sorption isotherms were measured using a porosity and surface area analyser (Micrometrics $3 \mathrm{Flex}$ ) at $77 \mathrm{~K}$. The samples were initially degassed overnight at $140{ }^{\circ} \mathrm{C}$ at approximately 0.2 mbar pressure. Prior to the sorption isotherm measurement, the samples were further degassed in-situ for 4 hours at $120^{\circ} \mathrm{C}$. The equivalent specific surface areas of the samples were determined using the Brunauer-Emmett-Teller (BET) method. ${ }^{79}$ The total pore volume was ascertained from the volume of $N_{2}$ adsorbed at a relative pressure $\left(P / P_{0}\right)$ of 0.97. The micropore volume was determined using the Dubinin-Radushkevich model. ${ }^{80}$

Low pressure gas sorption. Gas sorption tests at low pressure (up to 1 bar) were performed on a Micromeritics 3Flex sorption analyzer at $25^{\circ} \mathrm{C}$, using a water bath to control the temperature. The samples $(\sim 100 \mathrm{mg})$ were degassed overnight at $120^{\circ} \mathrm{C}$ at roughly $0.2 \mathrm{mbar}$ pressure and further degassed in situ for $4 \mathrm{~h}$ down to around $0.0030 \mathrm{mbar}$, before the start of the analysis. The gases were tested in the following order: nitrogen and carbon dioxide. The in situ degas step was repeated between each measurement.

High pressure gas sorption. Gas sorption tests at high pressures (up to 20 bar) were performed gravimetrically on an IGA-200 Intelligent gas analyzer from Hiden Isochema. The sample ( $\sim 30 \mathrm{mg})$ was added to a porous sample container, connected to a high-precision balance $( \pm 0.0001 \mathrm{mg})$. The sample was degassed in situ at $120^{\circ} \mathrm{C}$, using a furnace attachment, for $4 \mathrm{~h}$ before the start of the analysis. Ultrahigh vacuum of up to $10^{-6} \mathrm{mbar}$ can be created inside the chamber. Nitrogen and carbon dioxide sorption were performed at 
different temperatures $\left(10,25,40{ }^{\circ} \mathrm{C}\right)$, and the sample was degassed at $60{ }^{\circ} \mathrm{C}$ for $3 \mathrm{~h}$ between each run. The temperature was controlled by using a water/ethylene glycol bath.

\section{Computational model development}

The Grand Canonical Monte Carlo simulations ${ }^{60}$ for gas sorption in porous BNO were conducted using the $M e d e A^{\circledR} 2.22$ software suite ${ }^{61}$ developed by Materials Design Inc ${ }^{\circledR}$ and visualizations with VMD. ${ }^{81}$ The polymer consistent force field plus ( $\left.p c f f+\right)$ is employed with no modification. pcff+ is an all-atom force field based on the COMPASS ${ }^{82}$ framework which uses analytical forms to describe dispersion-repulsion interactions, point charges and classical bond stretching, bending and torsion terms. pcff+ is optimized to provide an accurate prediction of liquid properties and molecular conformations consistent with ab initio simulations. Fluid-fluid and fluid-solid interactions were assumed to be pairwise additive, where nonbonded interactions $\left(E_{v d w}\right)$ are calculated through a Mie-type term and the electrostatic contribution through a Coulombic function $\left(E_{C o u l}\right)$. For like atoms pairs we have:

$$
E_{v d W}=\epsilon\left[2\left(\frac{\sigma}{r}\right)^{9}-3\left(\frac{\sigma}{r}\right)^{6}\right]
$$

where $\epsilon$ is the potential depth in energy units and $\sigma$ corresponds to the molecular diameter in distance units. The Coulombic functions is defined by:

$$
E_{\text {Coul }}=\frac{C q_{i} q_{j}}{\epsilon_{c} r}
$$

where $C$ is an energy-conversion constant, $q_{i}$ and $q_{j}$ are the charges on the two atoms involved, and $\epsilon_{c}$ is the dielectric constant. In comparison with the usual Lennard-Jones 12-6 function, the repulsion region of equation (1) is softer with a higher attraction. For unlike atom pairs, a $6^{\text {th }}$ order combination rule is used to calculate the cross parameters between atoms $i$ and $j($ when $i \neq j)$ :

$$
\begin{gathered}
\sigma_{i j}=\left(\frac{\sigma_{i}^{6}+\sigma_{j}^{6}}{2}\right)^{1 / 6} \\
\epsilon_{i j}=2 \sqrt{\epsilon_{i} \epsilon_{j}}\left(\frac{\sigma_{i}^{3} \sigma_{j}^{3}}{\sigma_{i}^{6}+\sigma_{j}^{6}}\right)
\end{gathered}
$$


The cut-off radius is one half the box length. The electrostatic interactions are handled using Ewald summations using 7 vectors, with the same number of vectors in each direction, and a value of $\alpha=2.0$. The Ewald real space cut-off is applied between charge centres. The pcff+ forcefield parameters $(\sigma, \varepsilon)$ for the constituent $\mathrm{B}, \mathrm{N}$ and $\mathrm{O}$ atoms in boron oxynitride are: $\mathrm{BN}_{3}$ (3.4530 $\left.\AA, 0.0950 \mathrm{kcal} \mathrm{mol}^{-1}\right), \mathrm{NB}_{3}\left(3.3650 \AA, 0.1448 \mathrm{kcal} \mathrm{mol}^{-1}\right)$ and $\mathrm{OB}_{3}(3.3100 \AA, 0.1554$ $\left.\mathrm{kcal} \mathrm{mol}^{-1}\right)$. The partial charges for the atoms are: $\mathrm{B}(0.1000), \mathrm{N}(-0.1000)$ and $\mathrm{O}(-0.1000)$. The B-N and B-O bond lengths were $1.44 \AA$ and the bond angle was $120^{\circ}$. The boron oxynitride (BNO) nanoflake consisting of 24 atoms in total (12 boron atoms, 10 nitrogen atoms and 2 oxygen atoms) was initially designed as a non-periodic structure with the chemical composition tuned to the surface composition obtained through XPS measurements. The nanoflakes were subsequently ordered in a cell with a side dimension of $26.343 \AA$ (box volume of $18281 \AA^{3}$ ), avoiding overlapping of the BNO units. This structural realization was denoted as that at time $\mathrm{t}=0$ seconds in the following molecular dynamics simulations. No bridges were added between the structures and they were artificially fixed in space within the cell at time $t=0$ seconds. The final objective is to have the BNO nanoflakes randomly packed within the simulation box as a surrogate of the complex pore network and the amorphous/turbostratic morphology of porous BNO, as shown by Marchesini et al. ${ }^{34}$ through STEM tomography. To do so, molecular dynamics simulation in the canonical ensemble was performed employing LAMMPS $^{83}$ at an arbitrary high temperature $(3000 \mathrm{~K})$ with a timestep of $0.1 \mathrm{fs}$ over an arbitrary time period of $0-10000$ fs to ensure random placements of the flakes. The initialization step was conducted using 3-D periodicity with a non-bonded cutoff of $9.5 \AA$ and a long range precision of $0.00001 \AA$. Minimization was conducted using the conjugated gradient algorithm with a fast line search and maximum step of $0.05 \AA$. The attractive interactions between the nanoflakes were disabled to achieve random packing, whilst preventing agglomeration. $\mathrm{A}$ Nosé-Hoover thermostat ${ }^{84}$ was used to maintain the constant temperature requirement. The structures obtained from the molecular dynamics simulations were frozen in space and used as the templates for porous $\mathrm{BNO}$ adsorbents to simulate gas adsorption of $\mathrm{CO}_{2}, \mathrm{~N}_{2}$ and $\mathrm{CH}_{4}$ at low (< 1 bar) and high pressure $\left(0-20\right.$ bar) using the $M e d e A^{\circledR}$ Grand Canonical Monte 
Carlo ensemble. The pcff+ $(\sigma, \varepsilon)$ parameters for the adsorbates are: $\mathrm{CO}_{2}[\mathrm{C}(3.3000 \AA$, $\left.\left.0.0600 \mathrm{kcal} \mathrm{mol}^{-1}\right), \mathrm{O}\left(3.5258 \AA, 0.1040 \mathrm{kcal} \mathrm{mol}^{-1}\right)\right], \mathrm{N}_{2}\left[\mathrm{~N}\left(3.8008 \AA, 0.0598 \mathrm{kcal} \mathrm{mol}^{-1}\right)\right]$ and $\mathrm{CH}_{4}\left[\mathrm{C}\left(4.014 \AA, 0.0667 \mathrm{kcal} \mathrm{mol}^{-1}\right), \mathrm{H}\left(2.878 \AA, 0.0230 \mathrm{kcal} \mathrm{mol}^{-1}\right)\right]$. The partial charges for the atoms are: $\mathrm{CO}_{2}[\mathrm{C}(0.6650), \mathrm{O}(-0.3325)], \mathrm{N}_{2}[\mathrm{~N}(0.000), \mathrm{N}(0.000)]$ and $\mathrm{CH}_{4}[\mathrm{C}(-0.212)$ and $\mathrm{H}(0.053)]$. The bond lengths and bond angles in each adsorbate are: $\mathrm{CO}_{2}[\mathrm{C}=\mathrm{O}, 1.16 \AA$, $\left.180^{\circ}\right], \mathrm{CH}_{4}\left[\mathrm{C}-\mathrm{H}, 1.101 \AA, 107.66^{\circ}\right]$ and $\mathrm{N}_{2}\left[\mathrm{~N} \equiv \mathrm{N}, 1.0977 \AA, 180^{\circ}\right]$. The adsorption simulations were conducted using a grid LJ cutoff of $20.0 \AA$. The equilibration run was conducted for $2 x$ $10^{6}$ steps, with the pressure calculated using the virial formula, ${ }^{85}$ and the production run was conducted for $5 \times 10^{6}$ steps. The ratio of probabilities for displacement (rigid translations and rotations) moves to insertion moves was $0.3: 0.7$.

\section{References}

1. Gubbins, K. E.; Gu, K.; Huang, L.; Long, Y.; Mansell, J. M.; Santiso, E. E.; Shi, K.; ŚliwińskaBartkowiak, M.; Srivastava, D., Surface-Driven High-Pressure Processing. Eng. J. 2018, 4 (3), 311-320.

2. Xie, Z.; Su, B.-L., Crystalline Porous Materials: from Zeolites to Metal-Organic Frameworks (MOFs). Springer: 2020.

3. Ge, X.; Ma, S., $\mathrm{CO}_{2}$ Capture and Separation of Metal-Organic Frameworks. In Materials for Carbon Capture, Wiley: 2020; Vol. 3, pp 5-27.

4. Abbasi, Z.; Cseri, L.; Zhang, X.; Ladewig, B. P.; Wang, H., Metal-Organic Frameworks (MOFs) and MOF-Derived Porous Carbon Materials for Sustainable Adsorptive Wastewater Treatment. In Sustainable Nanoscale Engineering, Elsevier: 2020; pp 163-194.

5. Rodríguez-San-Miguel, D.; Montoro, C.; Zamora, F., Covalent Organic Framework Nanosheets: Preparation, Properties and Applications. Chem. Soc. Rev. 2020, 49 (8), 2291-2302.

6. Wang, Z.; Zhang, S.; Chen, Y.; Zhang, Z.; Ma, S., Covalent Organic Frameworks for Separation Applications. Chem. Soc. Rev. 2020, 49 (3), 708-735.

7. Qian, H.-L.; Yang, C.-X.; Yan, X.-P., Bottom-Up Synthesis of Chiral Covalent Organic Frameworks and their Bound Capillaries for Chiral Separation. Nat. Commun 2016, 7, 12104.

8. Jiang, L.; Gonzalez-Diaz, A.; Ling-Chin, J.; Roskilly, A.; Smallbone, A., Post-Combustion $\mathrm{CO}_{2}$ Capture from a Natural Gas Combined Cycle Power Plant Using Activated Carbon Adsorption. Appl. Energy 2019, 245, 1-15.

9. Yin, C. Y.; Aroua, M. K.; Daud, W. M. A. W., Review of Modifications of Activated Carbon for Enhancing Contaminant Uptakes from Aqueous Solutions. Sep. Purif. Technol. 2007, 52 (3), 403-415.

10. Shafeeyan, M. S.; Daud, W. M. A. W.; Houshmand, A.; Shamiri, A., A Review on Surface Modification of Activated Carbon for Carbon Dioxide Adsorption. J. Anal. Appl. Pyrol. 2010, 89 (2), 143151.

11. Liang, J.; Nuhnen, A.; Millan, S.; Breitzke, H.; Gvilava, V.; Buntkowsky, G.; Janiak, C., Encapsulation of a Porous Organic Cage into the Pores of a Metal-Organic Framework for Enhanced $\mathrm{CO}_{2}$ Separation. Angew. Chem. 2020, 132 (15), 6124-6129. 
12. Li, M.; Zhao, H.; Lu, Z.-Y., Porphyrin-Based Porous Organic Polymer, Py-POP, as a Multifunctional Platform for Efficient Selective Adsorption and Photocatalytic Degradation of Cationic Dyes. Microporous Mesoporous Mater. 2020, 292, 109774.

13. Zhang, S.; Taylor, M. K.; Jiang, L.; Ren, H.; Zhu, G., Light Hydrocarbon Separations Using Porous Organic Framework Materials. Chem. Eur. J. 2020, 26 (15), 3205-3221.

14. Kim, J.-J.; Lim, S.-J.; Ahn, H.; Lee, C.-H. J. M., Adsorption Equilibria and Kinetics of Propane and Propylene on Zeolite 13X Pellets. Microporous Mesoporous Mater. 2019, 274, 286-298.

15. Yan, B.; Yu, S.; Zeng, C.; Yu, L.; Wang, C.; Zhang, L., Binderless Zeolite NaX Microspheres with Enhanced $\mathrm{CO}_{2}$ Adsorption Selectivity. Microporous Mesoporous Mater. 2019, 278, 267-274.

16. Wang, S.; Peng, Y., Natural Zeolites as Effective Adsorbents in Water and Wastewater Treatment. J. Chem. Eng. 2010, 156 (1), 11-24.

17. Cao, D.; Lan, J.; Wang, W.; Smit, B., Lithium-Doped 3D Covalent Organic Frameworks: HighCapacity Hydrogen Storage Materials. Angew. Chem. Int. Ed. 2009, 48 (26), 4730-4733.

18. Connolly, B. M.; Madden, D. G.; Wheatley, A. E.; Fairen-Jimenez, D., Shaping the Future of Fuel: Monolithic Metal-Organic Frameworks for High-Density Gas Storage. J. Am. Chem. Soc. 2020, 142 (19), 8541-8549.

19. Li, H.; Li, L.; Lin, R.-B.; Zhou, W.; Zhang, Z.; Xiang, S.; Chen, B., Porous Metal-Organic Frameworks for Gas Storage and Separation: Status and Challenges. J. Energy Chem. 2019, 1 (1), 100006.

20. Attia, N. F.; Jung, M.; Park, J.; Jang, H.; Lee, K.; Oh, H., Flexible Nanoporous Activated Carbon Cloth for Achieving High $\mathrm{H}_{2}, \mathrm{CH}_{4}$, and $\mathrm{CO}_{2}$ Storage Capacities and Selective $\mathrm{CO}_{2} / \mathrm{CH}_{4}$ Separation. J. Chem. Eng. 2020, 379, 122367.

21. Weng, Q.; Wang, B.; Wang, X.; Hanagata, N.; Li, X.; Liu, D.; Wang, X.; Jiang, X.; Bando, Y.; Golberg, D., Highly Water-Soluble, Porous, and Biocompatible Boron Nitrides for Anticancer Drug Delivery. ACS Nano 2014, 8 (6), 6123-6130.

22. Lin, C.; Sun, K.; Zhang, C.; Tan, T.; Xu, M.; Liu, Y.; Xu, C.; Wang, Y.; Li, L.; Whittaker, A., Carbon Dots Embedded Metal Organic Framework@ Chitosan Core-Shell Nanoparticles for Vitro Dual Mode Imaging and pH-Responsive Drug Delivery. Microporous Mesoporous Mater. 2020, 293, 109775. 23. Wang, B.; Liu, X.; Gong, P.; Ge, X.; Liu, Z.; You, J., Fluorescent COFs with a Highly Conjugated Structure for Visual Drug Loading and Responsive Release. ChemComm 2020, 56 (4), 519-522.

24. Scicluna, M. C.; Vella-Zarb, L., Evolution of Nanocarrier Drug-Delivery Systems and Recent Advancements in Covalent Organic Framework-Drug Systems. ACS Appl. Nano Mater. 2020, 3 (4), 3097-3115.

25. Adebajo, M. O.; Frost, R. L.; Kloprogge, J. T.; Carmody, O.; Kokot, S., Porous Materials for Oil Spill Cleanup: a Review of Synthesis and Absorbing Properties. J. Porous Mater. 2003, 10 (3), 159-170. 26. Bhanja, P.; Modak, A.; Bhaumik, A., Porous Organic Polymers for $\mathrm{CO}_{2}$ Storage and Conversion Reactions. ChemCatChem 2019, 11 (1), 244-257.

27. Zeng, Y.; Zou, R.; Zhao, Y., Covalent Organic Frameworks for $\mathrm{CO}_{2}$ Capture. Adv. Mater. 2016, 28 (15), 2855-2873.

28. Ding, M.; Flaig, R. W.; Jiang, H.-L.; Yaghi, O. M., Carbon Capture and Conversion using MetalOrganic Frameworks and MOF-Based Materials. Chem. Soc. Rev. 2019, 48 (10), 2783-2828.

29. Drage, T. C.; Blackman, J. M.; Pevida, C.; Snape, C. E., Evaluation of Activated Carbon Adsorbents for $\mathrm{CO}_{2}$ Capture in Gasification. Energy Fuels 2009, 23 (5), 2790-2796.

30. Parlett, C. M.; Wilson, K.; Lee, A. F., Hierarchical Porous Materials: Catalytic Applications. Chem. Soc. Rev. 2013, 42 (9), 3876-3893.

31. Zhang, T.; Xing, G.; Chen, W.; Chen, L., Porous Organic Polymers: a Promising Platform for Efficient Photocatalysis. Mater. Chem. Front. 2020, 4 (2), 332-353.

32. Rogge, S. M.; Bavykina, A.; Hajek, J.; Garcia, H.; Olivos-Suarez, A. I.; Sepúlveda-Escribano, A.; Vimont, A.; Clet, G.; Bazin, P.; Kapteijn, F., Metal-Organic and Covalent Organic Frameworks as Single-Site Catalysts. Chem. Soc. Rev. 2017, 46 (11), 3134-3184. 
33. Yang, S.; Hu, W.; Zhang, X.; He, P.; Pattengale, B.; Liu, C.; Cendejas, M.; Hermans, I.; Zhang, X.; Zhang, J., 2D Covalent Organic Frameworks as Intrinsic Photocatalysts for Visible Light-Driven $\mathrm{CO}_{2}$ Reduction. J. Am. Chem. Soc 2018, 140 (44), 14614-14618.

34. Marchesini, S.; McGilvery, C. M.; Bailey, J.; Petit, C., Template-Free Synthesis of Highly Porous Boron Nitride: Insights into Pore Network Design and Impact on Gas Sorption. ACS Nano 2017, 11 (10), $10003-10011$.

35. Shankar, R.; Sachs, M.; Francàs, L.; Lubert-Perquel, D.; Kerherve, G.; Regoutz, A.; Petit, C., Porous Boron Nitride for Combined $\mathrm{CO}_{2}$ Capture and Photoreduction. J. Mater. Chem. A 2019, 7 (41), 23931-23940.

36. Shankar, R.; Marchesini, S.; Petit, C., Enhanced Hydrolytic Stability of Porous Boron Nitride via the Control of Crystallinity, Porosity and Chemical Composition. J. Phys. Chem. C 2019, 123 (7), 42824290.

37. Marchesini, S.; Regoutz, A.; Payne, D.; Petit, C., Tunable Porous Boron Nitride: Investigating its Formation and its Application for Gas Adsorption. Microporous Mesoporous Mater. 2017, 243, 154163.

38. Zhou, M.; Wang, S.; Yang, P.; Huang, C.; Wang, X., Boron Carbon Nitride Semiconductors Decorated with CdS Nanoparticles for Photocatalytic Reduction of $\mathrm{CO}_{2}$. ACS Catal. 2018, 8 (6), 49284936.

39. Weng, Q.; Wang, X.; Wang, X.; Bando, Y.; Golberg, D., Functionalized Hexagonal Boron Nitride Nanomaterials: Emerging Properties and Applications. Chem. Soc. Rev. 2016, 45 (14), 3989-4012.

40. Jiang, X.-F.; Weng, Q.; Wang, X.-B.; Li, X.; Zhang, J.; Golberg, D.; Bando, Y., Recent Progress on Fabrications and Applications of Boron Nitride Nanomaterials: a Review. J. Mater. Sci. Technol. 2015, 31 (6), 589-598.

41. Weng, Q.; Wang, X.; Bando, Y.; Golberg, D., One-Step Template-Free Synthesis of Highly Porous Boron Nitride Microsponges for Hydrogen Storage. Adv. Energy Mater. 2014, 4 (7).

42. Wang, D.; Xue, Y.; Wang, C.; Ji, J.; Zhou, Z.; Tang, C., Improved Capture of Carbon Dioxide and Methane via Adding Micropores Within Porous Boron Nitride Fibers. J. Mater. Sci. 2019, 54 (14), 10168-10178.

43. Jiang, H.; Ma, L.; Yang, Q.; Tang, Z.; Song, X.; Zeng, H.; Zhi, C., Three-Dimensional Porous Boron Nitride Foam for Effective $\mathrm{CO}_{2}$ Adsorption. Solid State Commun. 2019, 294, 1-5.

44. Lei, W.; Portehault, D.; Liu, D.; Qin, S.; Chen, Y., Porous Boron Nitride Nanosheets for Effective Water Cleaning. Nat. Commun 2013, 4, 1777.

45. Li, J.; Lin, J.; Xu, X.; Zhang, X.; Xue, Y.; Mi, J.; Mo, Z.; Fan, Y.; Hu, L.; Yang, X., Porous Boron Nitride with a High Surface Area: Hydrogen Storage and Water Treatment. Nanotechnology 2013, 24 (15), 155603.

46. Marchesini, S.; Wang, X.; Petit, C., Porous Boron Nitride Materials: Influence of Structure, Chemistry and Stability on the Adsorption of Organics. Front. Chem. 2019, 7, 160.

47. Song, Q.; Liang, J.; Fang, Y.; Guo, Z.; Du, Z.; Zhang, L.; Liu, Z.; Huang, Y.; Lin, J.; Tang, C., Nickel (II) Modified Porous Boron Nitride: An Effective Adsorbent for Tetracycline Removal from Aqueous Solution. J. Chem. Eng. 2020, 124985.

48. Weng, Q.; Kvashnin, D. G.; Wang, X.; Cretu, O.; Yang, Y.; Zhou, M.; Zhang, C.; Tang, D. M.; Sorokin, P. B.; Bando, Y., Tuning of the Optical, Electronic, and Magnetic Properties of Boron Nitride Nanosheets with Oxygen Doping and Functionalization. Adv. Mater. 2017, 29 (28), 1700695.

49. Xiao, F.; Chen, Z.; Casillas, G.; Richardson, C.; Li, H.; Huang, Z., Controllable Synthesis of FewLayered and Hierarchically Porous Boron Nitride Nanosheets. ChemComm 2016, 52 (20), 3911-3914.

50. Lu, X.; Zhang, M.; Jin, D.; Dang, Y.; Zhou, S.; Wei, S.; Zhu, H.; Zhao, L., Competitive Adsorption of $\mathrm{CO}_{2} / \mathrm{CH}_{4}$ in Porous Boron Nitride Nanomaterials. Mater. Lett. 2015, 161, 545-548.

51. Kayal, A.; Chandra, A., Water in Confinement between Nanowalls: Results for Hexagonal Boron Nitride versus Graphene Sheets from Ab Initio Molecular Dynamics. J. Phys. Chem. C 2019, 123 (10), 6130-6140. 
52. Srivastava, R.; Kommu, A.; Sinha, N.; Singh, J., Removal of Arsenic lons Using Hexagonal Boron Nitride and Graphene Nanosheets: a Molecular Dynamics Study. Mol. Simul. 2017, 43 (13-16), 985996.

53. Kommu, A.; Singh, J. K., Separation of Ethanol and Water Using Graphene and Hexagonal Boron Nitride Slit Pores: a Molecular Dynamics Study. J. Phys. Chem. C 2017, 121 (14), 7867-7880.

54. Cheng, J.; Zhang, L.; Ding, R.; Ding, Z.; Wang, X.; Wang, Z., Grand Canonical Monte Carlo Simulation of Hydrogen Physisorption in Single-Walled Boron Nitride Nanotubes. Int. J. Hydrog. Energy 2007, 32 (15), 3402-3405.

55. Mahdizadeh, S. J.; Tayyari, S. F., Methane Storage in Homogeneous Armchair Open-Ended Single-Walled Boron Nitride Nanotube Triangular Arrays: a Grand Canonical Monte Carlo Simulation Study. J. Mol. Model. 2012, 18 (6), 2699-2708.

56. Ahadi, Z.; Shadman, M.; Yeganegi, S.; Asgari, F., Hydrogen Adsorption Capacities of MultiWalled Boron Nitride Nanotubes and Nanotube Arrays: a Grand Canonical Monte Carlo Study. J. Mol. Model. 2012, 18 (7), 2981-2991.

57. Kumar, K. V.; Salih, A.; Lu, L.; Müller, E. A.; Rodríguez-Reinoso, F., Molecular Simulation of Hydrogen Physisorption and Chemisorption in Nanoporous Carbon Structures. Adsorpt. Sci. Technol. 2011, 29 (8), 799-817.

58. Di Biase, E.; Sarkisov, L., Systematic Development of Predictive Molecular Models of High Surface Area Activated Carbons for Adsorption Applications. Carbon 2013, 64, 262-280.

59. Salih, A.; Müller, E., Determination of Adsorptive and Diffusive Properties of Carbon DioxideMethane-Water in Coal-Like Structures Using Molecular Simulations. Nat. Preced. 2008, 1-1.

60. Smit, B., Grand Canonical Monte Carlo Simulations of Chain Molecules: Adsorption Isotherms of Alkanes in Zeolites. Mol. Phys. 1995, 85 (1), 153-172.

61. Yiannourakou, M.; Ungerer, P.; Leblanc, B.; Ferrando, N.; Teuler, J.-M., Overview of MedeA ${ }^{{ }_{-}}$ GIBBS Capabilities for Thermodynamic Property Calculation and VLE Behaviour Description of Pure Compounds and Mixtures: Application to Polar Compounds Generated from Ligno-Cellulosic Biomass. Mol. Simul. 2013, 39 (14-15), 1165-1211.

62. Kurakevych, O. O.; Solozhenko, V. L., Rhombohedral Boron Subnitride, B13N2, by X-Ray Powder Diffraction. Acta Crystallogr. C 2007, 63 (9), i80-i82.

63. Cholet, V.; Vandenbulcke, L.; Rouan, J.; Baillif, P.; Erre, R., Characterization of Boron Nitride Films Deposited from BCl 3-NH 3-H 2 Mixtures in Chemical Vapour Infiltration Conditions. J. Mater. Sci. 1994, 29 (6), 1417-1435.

64. Park, K.; Lee, D.; Kim, K.; Moon, D., Observation of a Hexagonal BN Surface Layer on the Cubic BN Film Grown by Dual Ion Beam Sputter Deposition. Appl. Phys. Lett 1997, 70 (3), 315-317.

65. Nazarov, A. S.; Demin, V. N.; Grayfer, E. D.; Bulavchenko, A. I.; Arymbaeva, A. T.; Shin, H. J.; Choi, J. Y.; Fedorov, V. E., Functionalization and Dispersion of Hexagonal Boron Nitride (h-BN) Nanosheets Treated with Inorganic Reagents. Chem. Asian J. 2012, 7 (3), 554-560.

66. Yan, Z.; Lin, J.; Yuan, X.; Song, T.; Yu, C.; Liu, Z.; He, X.; Liang, J.; Tang, C.; Huang, Y., Desulfurization of Model Oil by Selective Adsorption over Porous Boron Nitride Fibers with Tailored Microstructures. Sci. Rep. 2017, 7 (1), 3297.

67. Geick, R.; Perry, C.; Rupprecht, G., Normal Modes in Hexagonal Boron Nitride. Phys. Rev. 1966, 146 (2), 543.

68. Salih, A. Molecular Simulation of the Adsorption and Transport Properties of Carbon Dioxide, Methane, Water and their Mixtures in Coal-Like Structures. PhD Thesis, Imperial College London, 2010.

69. Sarkisov, L.; Harrison, A., Computational Structure Characterisation Tools in Application to Ordered and Disordered Porous Materials. Mol. Simul. 2011, 37 (15), 1248-1257.

70. Hyndman, R. J.; Koehler, A. B., Another Look at Measures of Forecast Accuracy. Int. J. Forecast. 2006, 22 (4), 679-688.

71. Sun, Q.; Li, Z.; Searles, D. J.; Chen, Y.; Lu, G.; Du, A., Charge-Controlled Switchable $\mathrm{CO}_{2}$ Capture on Boron Nitride Nanomaterials. J. Am. Chem. Soc 2013, 135 (22), 8246-8253. 

1999.

73. Los, J.; Kroes, J.; Albe, K.; Gordillo, R.; Katsnelson, M.; Fasolino, A., Extended Tersoff Potential for Boron Nitride: Energetics and Elastic Properties of Pristine and Defective hBN. Phys. Rev. B 2017, 96 (18), 184108.

74. Govind Rajan, A.; Strano, M. S.; Blankschtein, D., Ab Initio Molecular Dynamics and Lattice Dynamics-Based Force Field for Modelling Hexagonal Boron Nitride in Mechanical and Interfacial Applications. J. Phys. Chem. Lett 2018, 9 (7), 1584-1591.

75. Thiemann, F. L., Rowe, P., Müller, E.A., Michaelides, A, A Machine Learning Potential for Hexagonal Boron Nitride Applied to Thermally and Mechanically Induced Rippling. arXiv:2007.11448 2020

76. D'Alessandro, D. M.; Smit, B.; Long, J. R., Carbon Dioxide Capture: Prospects for New Materials. Angew. Chem. Int. Ed. 2010, 49 (35), 6058-6082.

77. Samanta, A.; Zhao, A.; Shimizu, G. K.; Sarkar, P.; Gupta, R., Post-Combustion $\mathrm{CO}_{2}$ capture Using Solid Sorbents: A Review. Ind. Eng. Chem. Res 2012, 51 (4), 1438-1463.

78. Shen, C.; Liu, Z.; Li, P.; Yu, J., Two-Stage VPSA Process for $\mathrm{CO}_{2}$ Capture from Flue Gas Using Activated Carbon Beads. Ind. Eng. Chem. Res 2012, 51 (13), 5011-5021.

79. Brunauer, S.; Emmett, P. H.; Teller, E., Adsorption of Gases in Multimolecular Layers. J. Am. Chem. Soc 1938, 60 (2), 309-319.

80. Chen, S.; Yang, R., Theoretical Basis for the Potential Theory Adsorption Isotherms. The Dubinin-Radushkevich and Dubinin-Astakhov Equations. Langmuir 1994, 10 (11), 4244-4249.

81. Humphrey, W.; Dalke, A.; Schulten, K., VMD: Visual Molecular Dynamics. J. Mol. Graph. Model. 1996, 14 (1), 33-38.

82. Sun, H., COMPASS: An Ab Initio Force-Field Optimized for Condensed-Phase Applications Overview With Details on Alkane and Benzene Compounds. J. Phys. Chem. B 1998, 102 (38), 73387364.

83. Plimpton, S., Fast Parallel Algorithms for Short-Range Molecular Dynamics. J. Comput. Phys. $1995,117,1-19$.

84. Hünenberger, P. H., Thermostat Algorithms for Molecular Dynamics Simulations. In Advanced Computer Simulation, Springer: 2005; pp 105-149.

85. Spycher, N. F.; Reed, M. H., Fugacity Coefficients of $\mathrm{H}_{2}, \mathrm{CO}_{2}, \mathrm{CH}_{4}, \mathrm{H}_{2} \mathrm{O}$ and of $\mathrm{H}_{2} \mathrm{O}-\mathrm{CO}_{2}-\mathrm{CH}_{4}$ mixtures: A Virial Equation Treatment for Moderate Pressures and Temperatures Applicable to Calculations of Hydrothermal Boiling. Geochim. Cosmochim. Acta 1988, 52 (3), 739-749. 